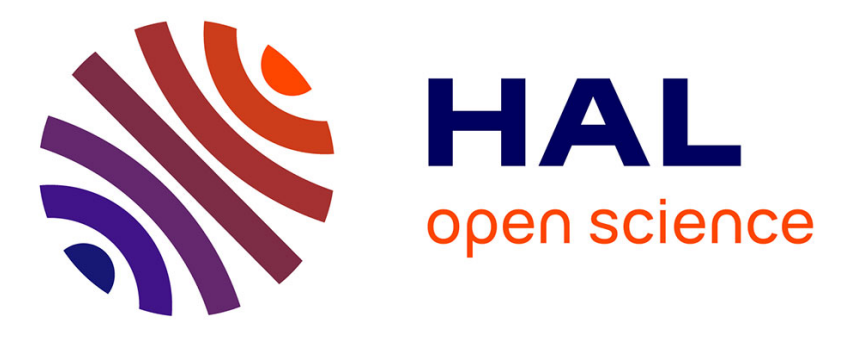

\title{
Surface acoustic wave depth profiling of a functionally graded material
}

Jozefien Goossens, Philippe Leclaire, Xiaodong Xu, Christ Glorieux, Loïc Martinez, Antonella Sola, Cristina Siligardi, Valeria Cannillo, Tom van Der Donck, Jean-Pierre Celis

\section{To cite this version:}

Jozefien Goossens, Philippe Leclaire, Xiaodong Xu, Christ Glorieux, Loïc Martinez, et al.. Surface acoustic wave depth profiling of a functionally graded material. Journal of Applied Physics, 2007, 102 (5), pp.053508. 10.1063/1.2774002 . hal-01326051

\section{HAL Id: hal-01326051 https://hal.science/hal-01326051}

Submitted on 3 Jun 2016

HAL is a multi-disciplinary open access archive for the deposit and dissemination of scientific research documents, whether they are published or not. The documents may come from teaching and research institutions in France or abroad, or from public or private research centers.
L'archive ouverte pluridisciplinaire HAL, est destinée au dépôt et à la diffusion de documents scientifiques de niveau recherche, publiés ou non, émanant des établissements d'enseignement et de recherche français ou étrangers, des laboratoires publics ou privés. 


\title{
Surface acoustic wave depth profiling of a functionally graded material
}

\author{
Jozefien Goossens, Philippe Leclaire, ${ }^{\text {a) }}$ Xiaodong $\mathrm{Xu},{ }^{\text {b) }}$ and Christ Glorieux ${ }^{\mathrm{c})}$ \\ Laboratorium voor Akoestiek en Thermische Fysica, Departement Natuurkunde en Sterrenkunde, \\ Katholieke Universiteit Leuven, PO 2416, Celestijnenlaan 200D, B-3001 Leuven, Belgium \\ Loic Martinez \\ Equipe Circuit Instrumentation et Modélisation en Electronique (ECIME) IUP GE, Université de Cergy, \\ Rue de Eragny, Neuville sur Oise, 95031 Cergy Pontoise Cedex, France \\ Antonella Sola, Cristina Siligardi, and Valeria Cannillo \\ Dipartimento di Ingegneria dei Materiali e dell'Ambiente, University of Modena and Reggio Emilia, \\ Via Vignolese 905, 41100 Modena, Italy \\ Tom Van der Donck and Jean-Pierre Celis \\ Departement Department of Metallurgy and Materials Engineering (MTM), Katholieke Universiteit Leuven, \\ PO 2450, Kasteelpark Arenberg 44, B-3001 Heverlee, Belgium
}

The potential and limitations of Rayleigh wave spectroscopy to characterize the elastic depth profile of heterogeneous functional gradient materials are investigated by comparing simulations of the surface acoustic wave dispersion curves of different profile-spectrum pairs. This inverse problem is shown to be quite ill posed. The method is then applied to extract information on the depth structure of a glass-ceramic (alumina) functionally graded material from experimental data. The surface acoustic wave analysis suggests the presence of a uniform coating region consisting of a mixture of $\mathrm{Al}_{2} \mathrm{O}_{3}$ and glass, with a sharp transition between the coating and the substrate. This is confirmed by scanning electron microscope with energy dispersive x-ray analysis.

\section{INTRODUCTION}

As material science and technology move forward, material structures are becoming more and more complex and delicate, increasing the need for accurate and nondestructive ways of characterizing them for quality evaluation, feedback for production improvement, or testing purposes. Here we address the specific issue of determining the compositional depth profile of functionally gradient materials (FGM). Taskspecific functionality of many materials is achieved by a surface treatment that upgrades the material properties at the surface and in subsurface regions. Already many researchers have addressed the challenge of characterizing materials with depth-depending subsurface properties by an accurate and nondestructive way. Along with the successful entry of laser ultrasonics in the field of nondestructive testing during the last three decades, Rayleigh wave spectroscopy has proven to be a powerful approach for characterizing materials with a compositional, and thus also elastic depth profile. ${ }^{1-4}$ Nowadays, using laser ultrasonics based technology, extremely high accuracy characterization of submicron coatings is possible. ${ }^{5}$

Rayleigh waves or surface acoustic waves (SAW) traveling on isotropic and uniform semi-infinite substrates are inherently nondispersive. The displacement and stress components of SAW contain terms that exponentially decay with depth. Roughly speaking the SAW penetration and thus also the probing depth is of the order of one wavelength. Thus, if the material is layered or inhomogeneous in the depth direction, then the short wavelength (or equivalently high frequency) wave components propagate at a velocity corresponding to the surface region of the material, while the long wavelength (or low frequency) components sense the material down to deeper regions. ${ }^{6}$ Therefore, for heterogeneous materials characterized by an elastic depth profile, the frequency dependence of the probing depth results in frequency dependence of the SAW propagation velocity, in other words velocity dispersion. At first sight, one might expect dispersion curves to be different for different elastic profiles, and that the inverse problem, ${ }^{7}$ i.e., the extraction of the elastic depth profile from the dispersion curve of the phase velocity, is well posed. If so, then SAW depth profiling should allow one to reconstruct elastic (and equivalently compositional) depth profiles of materials and e.g., to verify the details of a graded coating-substrate transition zone.

In this article, in view of the experimental case study of glass-ceramic graded coatings, we study the feasibility of SAW depth profiling for the determination of the glass graded coating thickness, and of the characteristics of the glass-ceramic transition zone, which are of importance for the overall performance of this functional gradient structure. In Sec. II, on the basis of simulations, we verify if different elastic profiles can yield the same dispersion curve, and thus if the inverse problem of SAW depth profiling is well-posed or ill-posed. In Sec. III, starting from the SAW velocity dispersion curve obtained by laser excited and detected Ray- 


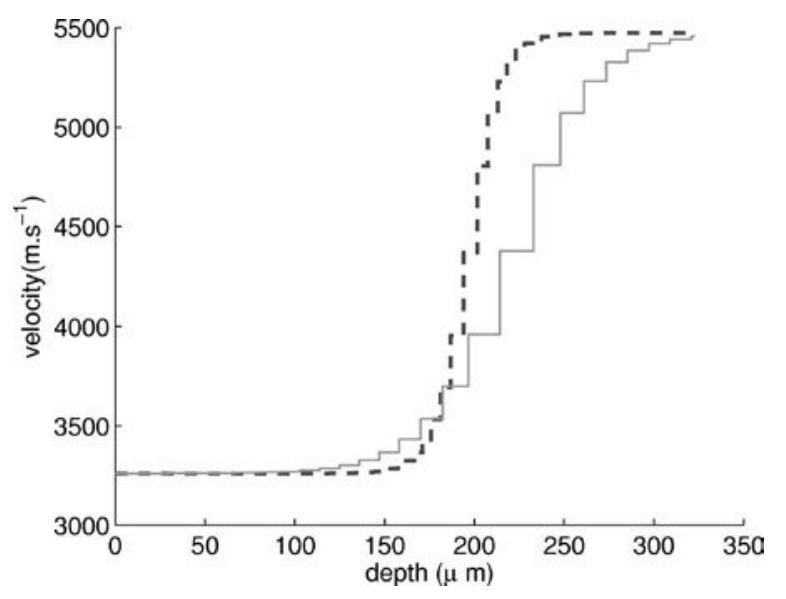

FIG. 1. Two examples (1: dashed lines, 2: full lines) of effective Rayleigh velocity profiles for materials with an $S$-type behavior of elastic properties. The elastic moduli used for the calculation of the dispersion curves in Fig. 2 were derived from the effective Rayleigh velocity by assuming a typical but arbitrary value for the Poisson's ratio and density.

leigh waves, and using an ad hoc coating-substrate model based on information from x-ray scanning electron microscope (SEM) compositional analysis, we extract and interpret elastic and compositional parameters of a glass-coated ceramic sample.

\section{A. FEASIBILITY OF SAW ELASTIC DEPTH PROFILING}

The issue of the feasibility of the inverse problem of the SAW depth profiling, i.e., the possibility of determining the elastic depth profile of a sample from the SAW dispersion curve, is inherently related with the existence of a one-to-one relation between profile and dispersion curve. In practical situations the criterion can be somehow reformulated. The inverse problem can be considered as ill posed, if sets of significantly different elastic profiles exist whose dispersion spectra are equal within the experimental error bar. If such profile-signal pairs are found, then one has to verify if the difference between different profiles "fitting" the same spectrum is significant or not, and if restricting the space of profiles under consideration by using a priori information avoids ambiguous cases.

Before tackling this issue though, one first has to solve the direct problem, i.e., calculating the frequency dependent SAW velocity $v_{\text {SAW }}(f)$, given the profiles of the elastic parameters [longitudinal velocity $v_{L}(z)$, transverse velocity $v_{T}(z)$, and density $\left.\rho(z)\right]$ in the depth direction $z$. We consider the typical case of one-dimensional (1D) propagation of SAW generated by a quasi-infinite line source, and traveling in the $x$ direction. One method to calculate the surface acoustic wave modes and velocities in such a structure is to discretize the continuous depth variation in the material and to consider it as a stratified material with characteristics slowly varying over the layers. This method, described in detail by Glorieux et al. ${ }^{6}$ is straightforward and equivalent with transfer matrix methods. ${ }^{8-10}$ These methods are usually simpler to implement than methods based on a Green's function approach. ${ }^{11-14}$ The accuracy can be set to any desired level, by choosing a sufficiently large number of layers.

For standard calculations we use 30 layers (Fig. 1). This is more than sufficient to describe smooth profiles, since the SAW velocity spectrum turns out not changed if, e.g., 50 layers are used. Note that the observation that differently discretized profiles yield quasi-indistinguishable dispersion spectra, is an indication of ill-posed character of SAW depth profiling. For the simulations in Fig. 1 we use more or less the same material and geometrical parameters as the ones used further on in the experimental section.

Glorieux et al. ${ }^{6}$ found that though isotropic materials are elastically described by their density and two elastic moduli, for calculating SAW dispersion curves, the profile of the "effective Rayleigh velocity" $v_{R \text {, eff }}(z)$ contains adequate and sufficient information to calculate the SAW dispersion curve $v_{\text {SAW }}(f)$. The effective Rayleigh velocity profile is the function describing the Rayleigh velocity value that is calculated by discretizing the material in virtual layers, and assigning to every layer a calculated Rayleigh velocity assuming that this layer is virtually semi-infinite. Stratified materials with, e.g., different density and Youngs modulus profiles, but with the same effective Rayleigh velocity profile yield quasi the same SAW velocity dispersion spectrum.

From their analytical solutions and physical nature it is not surprising that the phase velocity of the lowest order mode of Rayleigh waves in a stratified medium can be approximated $^{1}$ by a weighted integral of the velocity profile, with a weighing function that exponentially decays with a wavelength dependent, and thus frequency dependent, exponent. This behavior is similar to the one of thermal waves in photothermal experiments, where the penetration depth of the thermal diffusion modulations is given by the frequency dependent effective thermal diffusion length of the medium, and where information on the thermal conductivity profile of a heterogeneous material is extracted from the frequency dependence of the amplitude and phase of optically induced surface temperature oscillations. ${ }^{15}$ Provided some approximations and assumptions, the relation between profile and spectrum can be reduced to a linear set of equations that can be solved, e.g., by singular value decomposition (SVD). SVD studies show ${ }^{16}$ that the amount of reliable independent coefficients of the most relevant eigenvectors of the SVD matrix that are used to expand the profile and that can be reliably extracted is quite low. The number of reliable coefficients even decreases to less than 5 for only a few percent of uncertainty on the spectral data. In particular the deeper regions that are situated far from the surface of excitation and detection have a small influence on the signal and are difficult to extract from SVD analysis. Thus, unless one restricts the profiles to a strictly parametrized class, different profiles can yield photothermal spectra that are undistinguishable in the presence of experimental uncertainties, making the photothermal inverse problem partially ill posed. The similarity between the physics of the photothermal and SAW inverse problem suggests that the main conclusions concerning the photothermal inverse problem are also valid for SAW depth profiling. However, since the relation between the SAW velocity spectrum and the elastic profile of a material is difficult to analytically convert to a matrix equation, here we do not study the inverse problem of the SAW depth profiling 


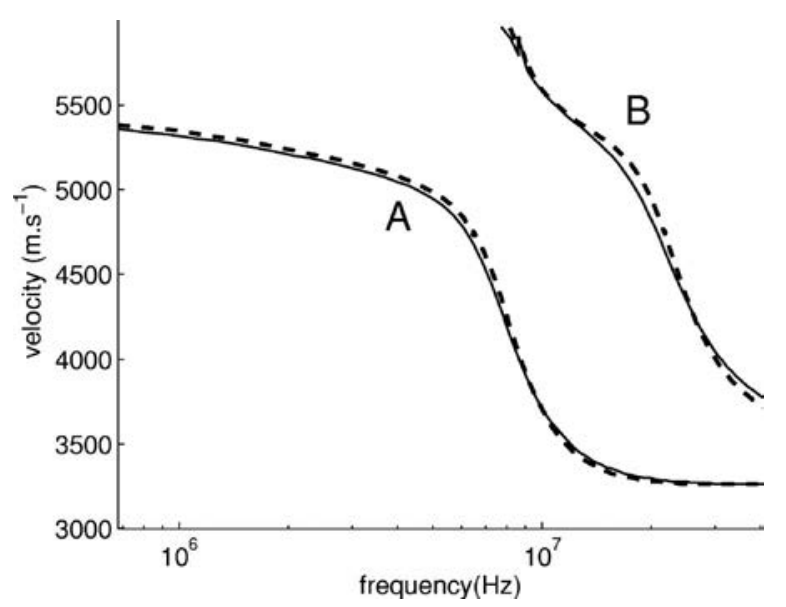

FIG. 2. Dispersion curves of the lowest $(A)$ and second lowest $(B)$ Rayleigh-type modes for a SAW traveling in the materials with elastic profiles 1 (dashed line) and 2 (full line) depicted in Fig. 1. The $S$-shaped curve $A$ varies from the effective Rayleigh velocity of the substrate at low frequencies toward the effective Rayleigh velocity of the top layer at high frequencies. Though profiles 1 and 2 are quite different, the corresponding SAW dispersion curves are almost coincident (see also Fig. 3 for a detailed procentual difference plot).

and judge the SAW inverse problem to be ill posed, if sets of significantly different elastic profiles can be found whose dispersion spectra are equal within the experimental error bar. If such profile-spectra pairs are found, then one has to verify if the difference between different profiles fitting the same spectrum is significant or not, and if restricting the space of profiles by using a priori information can lift the ambiguity.

Our search for such profiles was inspired by the experimental case study (Sec. III) of interest, where the investigated samples presumably consisted of a very thin quasiuniform glass top layer, an interdiffusion layer of glass and porous $(4.6 \%)$ ceramic, and a uniform "semi-infinite" (thickness much larger than longest SAW penetration depth) ceramic substrate. It is of technological interest not only to know the properties of the top layer and bulk substrate, but also to determine the width of the transition layer, and thus to characterize the detailed shape of the intermediate part of the $S$-shaped elastic depth profile.

For this kind of configuration, the high frequency part of a SAW dispersion curve always tends to the effective Rayleigh velocity $v_{R \text {,surf }}$ of the surface material. The asymptotic SAW velocity at low frequencies is the effective Rayleigh velocity $v_{R \text {,substr }}$ of the bulk substrate material. Thus the SAW dispersion curve varies between those two values in an $S$ shape, whose bending point is related to the thickness of the top layer. In the simulations in Fig. 2 we depict the influence of the details of the intermediate layer on the $S$-shape details of the dispersion curve. The results show that the dispersion curves of materials with quite different hyperbolic tangent effective Rayleigh velocity profiles are equal within $50 \mathrm{~m} \mathrm{~s}^{-1}$, a difference which is much smaller than the total velocity variation of the dispersion curves, and therefore is not easy to be resolved with experimental techniques. This is not only true for the lowest order Rayleigh mode, but unfortunately also for the second lowest.
This observation shows that essentially, in general, only the information on the bulk and surface value of the effective Rayleigh velocity, found from respectively the low and high frequency limit of the SAW velocity, gives completely unambiguous characteristics about the material, i.e., about the surface and the substrate limit of its effective Rayleigh velocity. If the search of an unknown profile is restricted to the limited parametrized profile space of hyperbolic tangent profiles, then also the inflection point of the $S$-shaped $v_{R \text {,eff }}(z)$ curve can be estimated quite well, since it is closely linked with the inflection point of the SAW dispersion curve. However, unless an actually not reachable high accuracy would be achieved on the experimental data, the information in the SAW dispersion curve on the slope at the inflection point is too strongly degenerated with small details in the shallow subsurface region of the profile. The slight difference between the two profiles in Fig. 1 in the shallow subsurface region between 120 and $180 \mu \mathrm{m}$ compensates the large opposite difference between 180 and $300 \mu \mathrm{m}$. This observation is consistent with the approximate Kernel integral expression for $v_{\mathrm{SAW}}(f)$ formulated by ${ }^{17}$ where in most of the frequency range shallow layers contribute considerably more to the integral than deeper regions.

We can conclude that unfortunately this phenomenon sets severe limits on the potential of SAW depth profiling, and makes the task of reliably extracting some relevant detailed information on the intermediate region not feasible in cases where a considerable amount of a priori information is not available. In particular, SAW depth profiling does not allow one to extract detailed information on the deeper parts of the glass-ceramic interdiffusion region of our sample, or of deep shape details of transition regions between a coating and substrate in general. In view of this, also earlier results in literature ${ }^{6}$ should be regarded with considerable prudence concerning the uncertainty on reconstructed profile details, if no a priori information was used.

\section{SAMPLE PREPARATION AND EXPERIMENTAL SETUPS}

The initial experimental aim of this work was to experimentally illustrate the feasibility of the SAW depth profiling for extracting detailed information about the compositional profile of functional gradient materials, e.g., for nondestructive evaluation of materials in technological applications. Graded glass-ceramic samples were chosen as test samples, since it was expected that they exhibit a nontrivial and gradual change in elastic properties with depth, therefore posing an interesting challenge for SAW depth profiling and extraction of subsurface material details from a surface measurement. In the previous section it was shown that there are considerable intrinsic limitations concerning this aspiration. In this section, we explain how the samples were produced and how the SAW velocity dispersion data were obtained experimentally. On the basis of the experimental data, we illustrate that the SAW dispersion curves on glass-graded ceramic samples can be obtained quite accurately, and that in spite of the earlier mentioned ill-posed character of the in- 
ertheless be effectively extracted, provided the use of some $a$ priori information.

The material used for the samples was alumina, which was infiltrated by glass. The purpose of putting a glass graded coating on a ceramic body is to improve the resistance to thermomechanical loading in daily use conditions and to protect the substrate against surface damage. ${ }^{18}$ The adhesion between glass and ceramic is in general quite good because of glass-ceramic interdiffusion during the production stage achieved by fusion of diffusing molten glass with the top layer of the slightly porous ceramic substrate. The quality of glass-coated ceramic samples is determined by the (uniformity of the) thickness of the top layer, and its adhesion to (and thus interdiffusion with) the ceramic substrate.

In order to produce the FGMs, a thin piece of glass (composition $39.78 \mathrm{~mol} \%$ of $\mathrm{CaO}, 4.52 \mathrm{~mol} \%$ of $\mathrm{ZrO}_{2}$, $55.70 \mathrm{~mol} \%$ of $\mathrm{SiO}_{2}$ ) of about $1 \mathrm{~mm}$ thick was put on a 5 $\mathrm{mm}$ thick, slightly porous $(4.6 \% \pm 0.6 \%$ porosity) alumina substrate $\left[\mathrm{Al}_{2} \mathrm{O}_{3}\right.$ from FN S.p.A. Nuove tecnologie e Servizi Avanzati. Bosco Marengo (AL), Italy] and subjected to a heat treatment which induced melting of the glass and infiltration into the alumina substrate. Two different heat treatments were applied: $2 \mathrm{~h}$ at $1873 \mathrm{~K}$ for sample I and $4 \mathrm{~h}$ at the same temperature for sample II. ${ }^{18}$ The small amount of residual glass that remained on the top surface was then polished away and the remaining surface was chromium coated by physical vapor deposition to ensure optimum conditions for absorbing the pump laser beam and to achieve specular reflection conditions for the probe laser beam during the SAW excitation and detection experiments. Care was taken during these after treatments not to change the sample characteristics by removing too much glass or even ceramic, or by heating the sample too much due to friction.

In the SAW experiments, Nd:YLF laser pulses with 1047 $\mathrm{nm}$ wavelength and $1 \mathrm{~mJ}$ energy were generated at a repetition rate of $1 \mathrm{kHz}$, and used to photoacoustically excite SAW in the thermoelastic regime. Two excitation geometries were used. In a first configuration, wideband SAW signals were generated focusing the pump laser beam to a Gaussian line of $6 \mathrm{~mm}$ long and $100 \mu \mathrm{m}$ wide, using a beam expander and a cylindrical lens. For most of the spectrum of the SAW signals the length of the excited line was considerably larger than the excited acoustic wavelengths. Therefore, and since moreover the SAW were detected on the symmetry axis in the near field region, in spite of some effect of diffraction on the wave amplitude, their propagation could be considered to be effectively one-dimensional as far as the analysis of the wave velocity is concerned. Theory shows ${ }^{19}$ that, concerning SAW propagation, a finite line can be considered as a combination of an (ideal) infinite line (1D SAW) and two point sources at the end of the line. As long as the line-probe distance (along the symmetry axis) is small compared with the end point-probe distance, the waves from the end points arrive much later and with much smaller amplitude (due to intrinsic geometrical attenuation of a point source) than the line-source induced 1D wave of interest.

In view of determining the frequency dependence of the SAW phase velocity an average was made of the repetitively recorded time dependence of the laser pulse excited displace- ment signals for a range of different pump-probe distances. Two detection schemes were used to obtain the signals. In a first setup, the out of plane displacement was recorded by an oscilloscope monitoring the signal from a Polytec ${ }^{\circledR}$ Laser Doppler Vibrometer with a cut-off frequency of $20 \mathrm{MHz}$. The sensitivity of the vibrometer was $50 \mathrm{~nm} \mathrm{~V}^{-1}$. The energy in the waves and the sensitivity of the detector were sufficiently high to allow reliable signal analysis between 3 and $20 \mathrm{MHz}$, corresponding with a SAW wavelength (and related penetration depth) between $0.2 \mathrm{~mm}$ and $1.5 \mathrm{~mm}$. The pump-probe distance was varied in 100 steps over $12 \mathrm{~mm}$ by moving the mount containing the mirrors and lens that were used to direct and focus the probe laser beam to a small spot on the sample surface.

In order to push the high frequency limit of the spectral signal content and reduce the SAW probing depth to just beneath the surface, thus reaching the saturated high frequency part of the SAW dispersion curve, we have also used a second setup that measured the angular deflection of a probe laser beam ${ }^{20}$ to detect the wave displacements. The upper frequency limit of such a setup is only limited by the bandwidth of the used photodetector (200 MHz in our case) and the focal width $x_{0}$ of the probe spot. We reduced $x_{0}$ to about $20 \mu \mathrm{m}$, allowing one to detect SAWs with wavelengths of the same order and longer. The corresponding maximum frequency content can be estimated as follows. ${ }^{21}$ If the excitation $I(x, t)=I_{0} \exp \left[-\left(t / t_{0}\right)^{2}\right] \exp \left[-\left(x / x_{0}\right)^{2}\right]$ is assumed to be Gaussian in time ( $1 / e$ duration $\left.2 t_{0}\right)$ and in space (1/e width $\left.2 x_{0}\right)$, corresponding with a spectrum $I(k, \omega)$ $=\left(t_{0} x_{0} / 4\right) \exp \left[-\left(\omega t_{0} / 2\right)^{2}\right] \exp \left[-\left(k x_{0} / 2\right)^{2}\right]$ with, inserting the wave dispersion relation $\mathrm{k}=\omega / \mathrm{v}_{\mathrm{SAW}}$, a $1 / e$ width $\Omega$ $=2 / \sqrt{\left[\left(t_{0}\right)^{2}+\left(x_{0} / \nu_{S A W}\right)^{2}\right]}$, then this leads to a temporal displacement signal $S(x, t)=S_{0} \exp \left\{-\left(1 / \sigma^{2}\right)\left[t-\left(x / \nu_{\mathrm{SAW}}\right)^{2}\right]\right\}$ with $\sigma^{2}=\left(t_{0}\right)^{2}+\left(x_{0} / \nu_{\mathrm{SAW}}\right)^{2}$. For $x_{0}=20 \mu \mathrm{m}$, this corresponds to a bandwidth of about $100 \mathrm{MHz}$.

In the used beam deflection setup SAW induced surface slope variations deflecting the probe beam resulted in a change of light intensity balance between the two parts of the beam which were obtained by splitting the laser beam into two more or less equal parts using two mirrors in a knife edge configuration. Using a lens, the two parts of the beam were focused on 2 respective photodiodes. Their difference signal, proportional with the slope change of the sample surface, was sent to the oscilloscope. For reasons of signal stability, while scanning the pump-probe distance, the probe laser spot was kept at a fixed position on the sample surface, optimized for optimum optical quality and reflectivity. Scanning the probe beam would require also to scan the beamsplitting deflection mirrors, lenses, and detectors, ${ }^{22}$ which would introduce large, sample roughness induced, dc slope fluctuations, rendering the balance of the differential photodetector and thus its sensitivity very unstable. Therefore, instead, the mirror and lens directing and focusing the pump laser beam to a line on the sample were scanned in 100 steps of $100 \mu \mathrm{m}$ over a range of $10 \mathrm{~mm}$.

Though with laser excitation and detection with respectively a line source and beam deflection quite high frequency waves can be analyzed, the technique is still somehow lim4 ited by practical limitations concerning how sharp the pump 


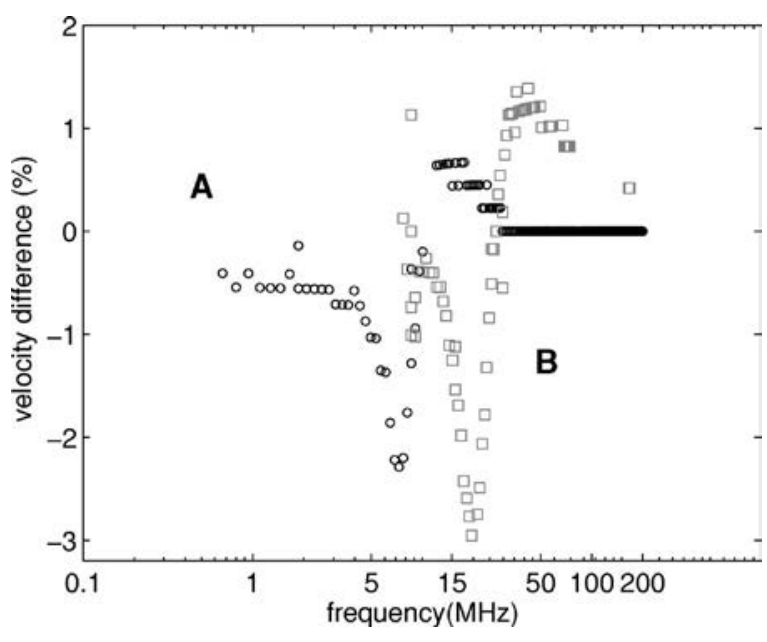

FIG. 3. Procentual difference between the SAW dispersion curves of the materials with profiles 1 and 2 shown in Fig. 1 , for the lowest ( $A$, circles) and second lowest ( $B$, squares) SAW modes. In spite of the significant difference between the profiles, the difference between the respective dispersion curves is only a few percent. Apparently large profile differences in deep regions can be roughly compensated by differences in more shallow regions. This observation indicates the significant ill-posed character of the inverse problem of depth profiling of elastically inhomogeneous materials.

and probe beam can be focused on the sample. Only when the excitation and detection regions are narrower than a wavelength, the corresponding spectral component of the wave can be easily detected. In practice one cannot make the laser spots smaller than the optical diffraction limit, and, more relevant for our case, than the threshold focal width above which the laser energy starts to induce surface damage by melting and ablation. A convenient alternative to push the bandwidth of experimental beam deflection signals to higher frequency limits without causing surface damage is a grating excitation scheme. In this configuration, ${ }^{23}$ by making use of a diffractive optic (a so-called phase mask), the laser beam generated by a pump laser was split in two parts, which, using a pair of lenses, were then collimated and finally crossed again on the sample surface under an angle. In this way, due to optical interference and via thermoelastic excitation, a spatially periodic wave packet with a short repetition distance of $70 \mu \mathrm{m}$, and thus temporal burst signals with high frequency content, was excited. Since the optically deposited thermoelastic energy is selectively concentrated in a narrow-band signal, while neither probe or pump laser beam need to be strongly focused on the sample surface, this configuration allows one to efficiently excite and detect short wavelengths and high frequencies without risk for surface damage due to high laser beam intensities. Alternatively, as in the case of line excitation, the grating excited wave propagation was also analyzed by monitoring the beam deflection signal while scanning the pump-probe distance.

\section{EXPERIMENTAL RESULTS AND DISCUSSION}

The spatiotemporal dependence of the optical beam deflection signals excited by a line source is shown in Fig. 4. Besides the Rayleigh wave, also the Scholte wave, which travels at the speed of sound in air, can be clearly distinguished. The Rayleigh wave packet broadens with increasing

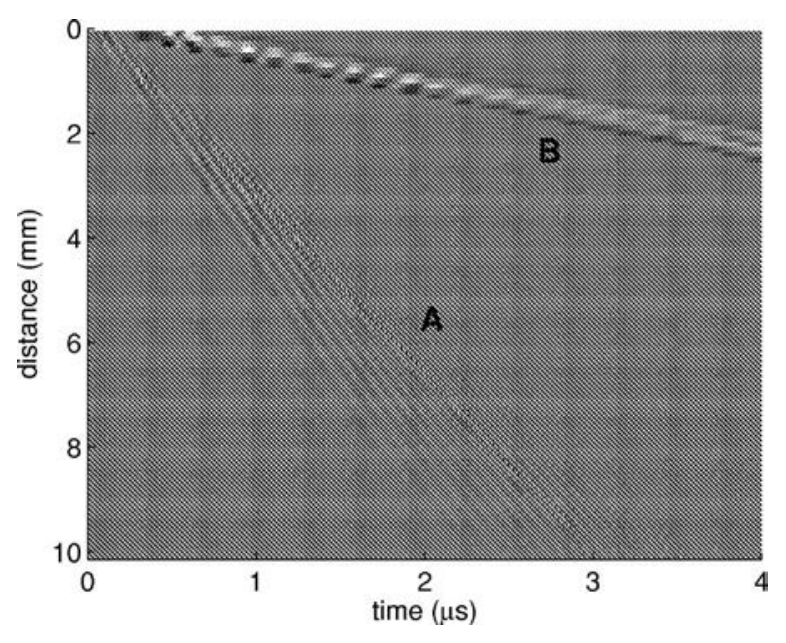

FIG. 4. Spatio-temporal dependence of SAW displacement signals detected by optical beam deflection. The broadening of the Rayleigh mode wave packet $(A)$ reveals dispersive behavior, due to the inhomogeneous elastic depth profile in the glass-ceramic functional gradient sample. The Scholte wave $(B)$, detected via refractive index changes in the air region trespassed by the probe laser beam, travels nondispersively at the sound velocity of air.

pump-probe distance, revealing dispersion due to the presence of an inhomogeneous elastic depth profile. For the sake of quantitative, frequency resolved analysis of the phase velocity, a numerical two-dimensional (2D) Fourier transform of the $S(x, t)$ matrix was taken, thus obtaining $S(k, \omega)$, with $k$ the wave number, $\omega=2 \pi f$ the angular frequency and $f$ the frequency. In order to avoid fast Fourier transform (FFT) artifacts, before transforming the data, the mean of the signals was substracted, and the relevant part of the signals was windowed with a smooth windowing function.

In the $|S(k, \omega)|$ diagram, as illustrated in Fig. 5, $(k, \omega)$ pairs on the Rayleigh mode can be easily distinguished, lead-

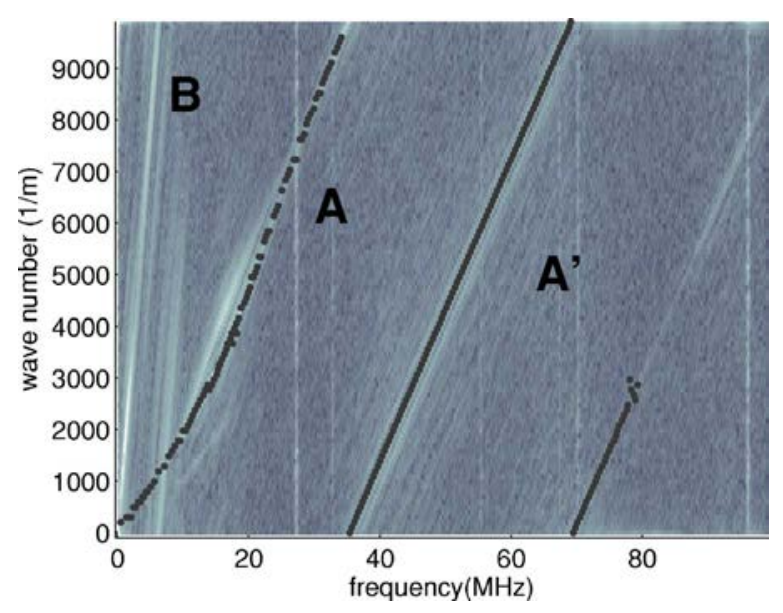

FIG. 5. Two-dimensional map of the Fourier transform amplitude $|S(\omega, k)|$ in frequency-wave number domain of the line source excited SAW displacement signals shown in Fig. 4. Different wave modes are characterized by their $k, \omega$ curve. Via the relation $v(f)=\omega / k$ the phase velocity of the Rayleigh $(A)$ and the Scholte mode $(B)$ can be determined. Due to spatial undersampling, the parts $\left(A^{\prime}\right)$ of the dispersion curve for wave numbers higher than half of the sampling wave number $k_{\max } / 2$ are folded back to low wave numbers by multiples of $k_{\max } / 2$. By unwrapping them, the data can be reliably analyzed until $100 \mathrm{MHz}$. The dotted curves indicate fits of the dispersion curve whose points were determined by performing a maximum 5 


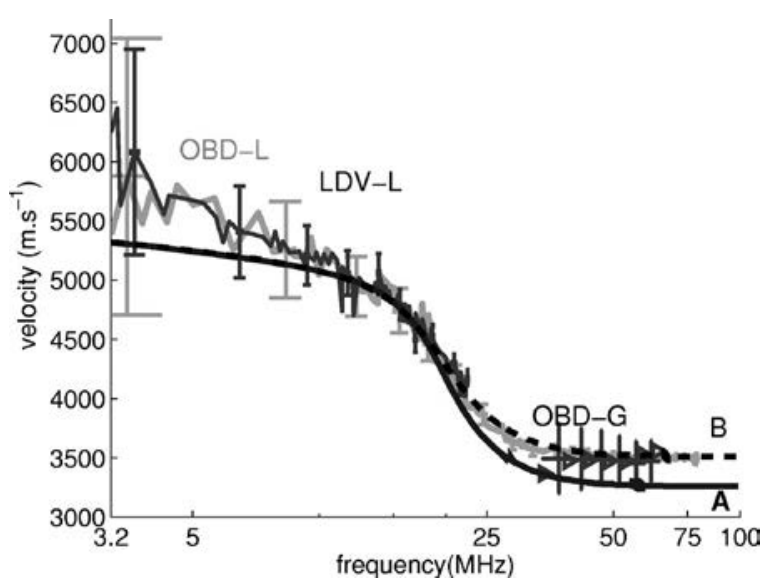

FIG. 6. Frequency dependence of the SAW velocity derived in different ways. Optical beam deflection (OBD-L: thin lines, line source excitation) and laser Doppler (LDV-L: bold gray lines) detection obtained for sample I were used to detect the spatiotemporal dependence of a Gaussian wave packet generated by focusing a pulsed pump laser beam to a Gaussian line on the sample. The dispersion curve of the phase velocity was determined from the $k, \omega$ amplitude spectrum of the displacement signals, as shown, e.g., in Fig. 5. Also grating excitation geometry (G, triangles) was used, by crossing two pulsed pump laser beams on the sample surface, in combination with detection by optical beam deflection. The experimental data can clearly not be fitted by simulation $A$, which assumes a pure glass top layer on top of a pure ceramic substrate, but are well fitted by simulation $B$, which assumes ad hoc elastic properties of a glass-ceramic mixture for the top layer, which were chosen so as to achieve a good fit for the high frequency limit of the SAW velocity (see also Fig. 7). The horizontal and vertical error bars were determined from the uncertainty on the frequency and the wave vector. The experimental dispersion curve for sample II was found equal within the error bar and for the sake of clarity is therefore not shown.

ing to $k(\omega)$ dispersion relations. The slightly $S$-shaped form of the fitted curve connecting the maxima $k_{\max }(f)$ in the amplitude diagram $|S(k, f)|$ confirms the presence of dispersion. The frequency dependence of the phase velocity $v_{\mathrm{SAW}}(\omega)$, summarized in Fig. 6 for data obtained from measurement results of laser Doppler vibrometer and optical beam deflection in line excitation mode and optical beam deflection in grating excitation mode, was determined using the relation $v_{\mathrm{SAW}}(\omega)=\omega / k_{\max }(\omega)$, with $\omega=2 \pi f$. Because there was not enough difference between the curves to discriminate between the two samples, only experimental curves for sample I are shown.

The laser Doppler vibrometer data were found reliable till about $30 \mathrm{MHz}$, which is somewhat higher than $20 \mathrm{MHz}$, the cut-off frequency stated by the manufacturer of the instrument. They are consistently overlapped and extended by the beam deflection data to $100 \mathrm{MHz}$, both in line excitation and in grating excitation mode. As mentioned earlier, in the latter mode of operation, a spatially periodic SAW burst with wavelength content centered around $\lambda=70 \mu \mathrm{m}$ was analyzed. Maximum amplitudes in the $A(k, \omega)$ map were found around $50 \mathrm{MHz}$, in the form of a stretched island, which overlapped very well with the corresponding part of the dispersion curve determined with line excitation.

Note that in all mentioned pump-probe scanning schemes the excitation and probe geometry only affect the bandwidth. The details of the transfer function of the chosen excitation-detection combinations do not affect the found frequency-wave vector pairs that are used to determine the
TABLE I. Material characteristics.

\begin{tabular}{lcc}
\hline \hline & Glass & Ceramic \\
\hline Longitudinal velocity $\left(\mathrm{m} \mathrm{s}^{-1}\right)$ & $6780( \pm 100)$ & $9840( \pm 300)$ \\
Shear velocity $\left(\mathrm{m} \mathrm{s}^{-1}\right)$ & $3500( \pm 30)$ & $6000( \pm 100)$ \\
Rayleigh velocity $\left(\mathrm{m} \mathrm{s}^{-1}\right)$ & $3260( \pm 30)$ & $5480( \pm 30)$ \\
Density $\left(\mathrm{kg} \mathrm{m}^{-3}\right)$ & $2970( \pm 20)$ & $3800( \pm 40)$ \\
\hline \hline
\end{tabular}

velocity dispersion curve. The reason is that while scanning, the transfer function is kept invariant and that for the 2D Fourier analysis, only spectral changes due to the increasing travel time and distance are of importance.

Interestingly, using grating excitation, the velocities of the spatially periodic wave modes packets can also be determined by taking a Fourier transform of the signal and then multiplying the dominant frequencies (maxima in amplitude spectrum) with the excited wavelength. The wavelength of the excited waves can be controlled by optically tuning the crossing angle of the excitation beams. It can be calibrated to high precision by making use of a reference sample with known Rayleigh velocity.

Joining all experimental results, the spectrum is covered from the low frequency regime where the coating is acoustically thin, till the high frequency regime, where the dispersion curve is dominated by the elastic properties of the coating only. The error bars indicated in Fig. 6 were derived from the finite discretization step $\Delta k$ due to the finite scanning range $\left(x_{\max }-x_{\min }=12 \mathrm{~mm}\right)$, and the finite discretization step $\Delta \omega$ due to the finite acquisition time range $\left(t_{\max }-t_{\min }\right.$ $=16 \mu \mathrm{s}$ ), using classical error propagation of the expression $v=\omega / k$. The uncertainty related to the frequency discretization due to the finite length of the recorded time signals, was negligible. For example at $5 \mathrm{MHz}$, the error is about $10 \%$

$$
\begin{aligned}
\frac{\Delta v}{v} & =\frac{\Delta k}{k}=\frac{v_{\mathrm{SAW}} / f}{x_{\max }-x_{\min }}=\frac{5500 \mathrm{~m} / \mathrm{s}}{5 \mathrm{MHz} 12 \mathrm{~mm}} \\
& =10 \% \cong \frac{5500 \mathrm{~m} \mathrm{~s}^{-1}}{6000 \mathrm{~m} \mathrm{~s}^{-1}} .
\end{aligned}
$$

Unfortunately, reducing this uncertainty by increasing the scanning range is difficult to achieve in practice.

In Fig. 6 the experimental dispersion data for sample I are also compared with two simulated curves that are based on a priori knowledge and assumptions about the sample properties (Table I). At low frequencies the difference between experimental data and simulations lies within the error bar. The experimental uncertainty is large because the SAW wavelengths are quite large with respect to the total scanning distance. The low frequency range is also somewhat less reliable because of diffraction effects due to the finite length of the source compared to the acoustic wavelength.

As explained in Sec. II, given the finite accuracy on the experimental phase velocity dispersion curve, the inverse problem of SAW depth profiling is only feasible if a priori knowledge is used about the sample structure. In particular, the information in $v_{R}(f)$ about the exact shape of the transition range between the top and substrate region is quite am6 
experimental data, a simple two-layer model was used. The used elastic parameters of the glass top layer (with best fitting thickness of $210 \mu \mathrm{m}$ ) and the ceramic substrate (Table I) were taken from independent SAW and bulk acoustic wave measurements on bulk ceramic and bulk glass samples from the same production batch. The SAW measurements were done using scanning laser Doppler vibrometry as for the glass-ceramic samples. The longitudinal and shear velocity of the substrate materials were obtained by pulse-echo measurements by use of $10 \mathrm{MHz}$ piezoelectric shear and longitudinal transducers. The corresponding Rayleigh velocity values for glass and ceramic were derived from the latter values and found consistent with the values from the SAW measurement (see Table I). The densities of the glass and ceramic materials were derived from the measured volume and mass of pure ceramic and glass samples. The values are similar to the ones in Ref. 18 for similar samples.

When comparing the simulated dispersion curve to the experimental results, it becomes clear that the high frequency limit $(100 \mathrm{MHz})$ of the experimental dispersion curve on the graded glass-coated ceramic samples $\left(3500 \mathrm{~m} \mathrm{~s}^{-1}\right)$, tends to a $200 \mathrm{~m} \mathrm{~s}^{-1}$ higher value than the Rayleigh velocity of pure glass $\left(3260 \mathrm{~m} \mathrm{~s}^{-1}\right)$, the assumed top layer material in the simulation. This difference is significantly larger than the measurement uncertainty $\left(20 \mathrm{~m} \mathrm{~s}^{-1}\right)$ of the beam deflection data with line excitation in that frequency range, and thus requires further explanation. For this reason, we show a second simulation, in which the elastic properties of the top layer (mainly its shear velocity) were adjusted in order to fit the experimental dispersion curve. This yielded a good fit, with a value for the effective Rayleigh velocity of $3500 \mathrm{~m} \mathrm{~s}^{-1}$ for the top layer, and a thickness of $210 \mu \mathrm{m}$. This observation reveals that the top layer of the sample, i.e., the range between $10 \mu \mathrm{m}$, the shortest probed depth at 100 $\mathrm{MHz}$, and $210 \mu \mathrm{m}$, the fitted transition depth, does not consist of pure glass as initially expected. Its velocity value is higher, suggesting a mixed composition glass and ceramic. This assumption was found to be consistent with scanning electron microscope with energy dispersive $\mathrm{x}$-ray (SEMEDX) analysis of a cross section of the sample that were taken a posteriori. These revealed that the initial thin glass top layer had been completely removed during the polishing procedure that was performed in preparation of the SAW analysis. The micrographs also clarified that the sample was effectively a 2-layer sample, with a uniform top layer of ceramic fully interdiffused by glass and a uniform, glass-free substrate. The situation was thus different than the one for the similar materials investigated in Ref. 18, where a gradual composition transition was found. Interestingly, the experimental dispersion curve for sample II was found equal within the error bar with the one of sample I, suggesting that for our samples the additional duration of the heat treatment did not induce additional glass diffusion, so that the sample structure remained unchanged. It is thus not surprising that the experimental data are fitted well by a 2-layer model simulation. Moreover, keeping in mind the earlier mentioned degeneracy of information concerning different profile details, it would not be useful to fit the experimental data with a more sophisticated model consisting of a uniform top layer, a smooth

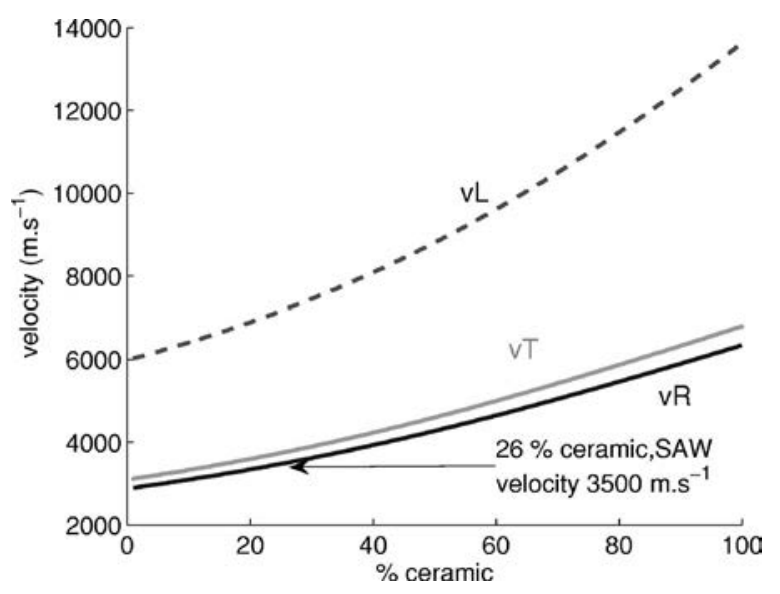

FIG. 7. Using the model of Berryman (Refs. 24 and 25), the value of $3500 \mathrm{~m} \mathrm{~s}^{-1}$, found for the top region of the sample, yields a $26 \%$ mass percentage of glass.

transition region, and uniform bulk material, since transition layer information is very difficult to extract.

Using the model of Berryman ${ }^{24,25}$ for elastic properties of composite materials, the value of $3500 \mathrm{~m} \mathrm{~s}^{-1}$ for the Rayleigh velocity of the surface layer corresponds to a effective average composition with $26 \%$ mass percentage (or equivalently $20 \mathrm{~mol} \%$ ) of glass. The determination of this value is illustrated in Fig. 7, where, using the elastic parameters of pure glass and pure ceramic in Table I and using the model of Berryman, the theoretical longitudinal velocity, the theoretical shear velocity, and the thereof deduced Rayleigh velocity are plotted versus the glass concentration in a glassceramic compound. Interestingly, the found composition of the top layer reveals the presence of much more glass than one could perhaps expect on the basis of the bulk porosity $(4.6 \%)$ of the ceramic (assuming that all the glass has diffused into the ceramic substrate). This was confirmed by SEM-EDX analysis, which revealed even more than $50 \mathrm{~mol} \%$ of glass. This is significantly more than the one obtained by SAW analysis, not surprisingly indicating that Berrymans model, ${ }^{24,25}$ that was constructed for specific geometries of heterogeneity, cannot be used in a quantitative way for this sample.

It thus seems that due to the high temperatures during the fusion treatment, and enhanced by the low melting point of the ternary mixture of $\mathrm{SiO}_{2}, \mathrm{CaO}$, and $\mathrm{Al}_{2} \mathrm{O}_{3}$, there has occurred a chemically driven mutual interdiffusion of $\mathrm{SiO}_{2}$ and $\mathrm{CaO}$ from the glass top layer into the substrate on one hand and $\mathrm{Al}_{2} \mathrm{O}_{3}$ from the ceramic substrate into the top layer on the other hand, finally resulting in a uniform mixed top layer. This intermixing is actually quite favorable for the mechanical adhesion properties of the resulting composite structure.

\section{CONCLUSION}

Simulations of SAW dispersion curves for materials with different inhomogeneous elastic depth profiles show that details of the transition zone of interdiffused coating-substrate samples can easily remain invisible in the SAW dispersion 7 curve due to a mixed influence of dominant shallow features 
and weakly affecting deep features of the profile on the wave propagation. This severely limits the potential of SAW depth profiling. However, in practice, this difficulty can often be circumvented by using available a priori information about the profile. Moreover, SAW depth profiling does allow one to extract other important profile parameters.

Experimental SAW dispersion curves obtained with a laser Doppler vibrometer and with a beam deflection setup on glass-graded ceramic samples were found to be consistent. The two techniques were shown to be complementary and suitable for SAW depth profiling in the $\mu \mathrm{m}$-mm depth range. Also experimental results line and grating source excitation gave consistent values for the SAW velocity.

The high frequency limit of the experimental data on glass-ceramic samples revealed a mixed composition of the surface region, which was confirmed by SEM-EDX analysis. In spite of to some extent intrinsic limitations concerning details in the transition region between the top layer and substrate of $S$-type structures, the experimental application of SAW spectroscopy was shown to be suitable for extracting useful information on the elastic and composition profile of functionally gradient materials in a noncontact and nondestructive way.

\section{ACKNOWLEDGMENTS}

The authors would like to thank K. U. Leuven, Belgium (GOA project 2002/04 and GOA project 2007/06/TBA) and FWO-V (research project LUFOGLAV G.0125.03 and research credit to C. Glorieux 1.5.124.05) for financial support of this research.
${ }^{1}$ T. L. Szabo, J. Appl. Phys. 46, 1448 (1975).

${ }^{2}$ B. R. Tittmann, L. A. Ahlberg, J. M. Richardson, and R. B. Thompson, IEEE Trans. Ultrason. Ferroelectr. Freq. Control 34, 500 (1987).

${ }^{3}$ G. A. Gordon and B. R. Tittmann, Rev. Prog. Quant. Nondestr. Eval. 6, 595 (1994)

${ }^{4}$ G. A. Gordon and B. R. Tittmann, Rev. Prog. Quant. Nondestr. Eval. 15, 1597 (1996).

${ }^{5}$ A. A. Maznev, A. Mazurenko, L. Zhuoyun, and M. Gostein, Rev. Sci. Instrum. 74, 667 (2003).

${ }^{6}$ C. Glorieux, W. Gao, S. E. Kruger, K. Van de Rostyne, W. Lauriks, and J. Thoen, J. Appl. Phys. 88, 4394 (2000).

${ }^{7}$ A. Wirgin, Wavefield Inversion (Springer, Vienna, 1999), pp. 241-304.

${ }^{8}$ Th. Pastureaud, V. Laude, and S. Ballandras, Appl. Phys. Lett. 80, 2544 (2002).

${ }^{9}$ E. L. Adler, IEEE Trans. Ultrason. Ferroelectr. Freq. Control 37, 485 (1990).

${ }^{10}$ A. Cheng, T. W. Murray, and J. D. Achenbach, J. Acoust. Soc. Am. 110, 848 (2001).

${ }^{11}$ O. Matsuda and C. Glorieux, J. Acoust. Soc. Am. 121, 3437 (2007).

${ }^{12}$ J. P. Groby, L. De Ryck, Ph. Leclaire, A. Wirgin, W. Lauriks, R. Gilbert, and Y. Xuh, Math. Methods Appl. Sci. 30, 91 (2007).

${ }^{13}$ E. L. Tan, IEEE Trans. Ultrason. Ferroelectr. Freq. Control 49, 929 (2002).

${ }^{14}$ L. De Ryck, J. P. Groby, P. Leclaire, W. Lauriks, A. Wirgin, Z. E. A. Fellah, and C. Depollier, Appl. Phys. Lett. 90, 181901 (2007).

${ }^{15}$ C. Glorieux and J. Thoen, J. Appl. Phys. 80, 6510 (1996).

${ }^{16} \mathrm{C}$. Glorieux, R. Li Voti, J. Thoen, M. Bertolotti, and C. Sibilia, Inverse Probl. 15, 1149 (1999).

${ }^{17}$ D. Colton and R. Kress, Integral Equation Methods in Scattering Theory (Wiley, New York, 1983).

${ }^{18}$ V. Cannillo, T. Manfredini, C. Siligardi, and A. Sola, J. Eur. Ceram. Soc. 26, 2685 (2006).

${ }^{19}$ A. Mourad, C. Desmet, and J. Thoen, Ultrasonics 35, 393 (1997).

${ }^{20}$ A. Neubrand and P. Hess, J. Appl. Phys. 71, 227 (1992).

${ }^{21}$ C. B. Scruby and L. E. Drain, Laser Ultrasonics: Techniques and Applications (Adam Hilger, Bristol, United Kingdom, 1990).

${ }^{22}$ C. Desmet, V. Gusev, W. Lauriks, C. Glorieux, and J. Thoen, Opt. Lett. 22, 69 (1997).

${ }^{23}$ A. A. Maznev, K. A. Nelson, and J. A. Rogers, Opt. Lett. 23, 1319 (1998).

${ }^{24}$ J. G. Berryman, J. Acoust. Soc. Am. 68, 1809 (1980).

${ }^{25}$ J. G. Berryman, J. Acoust. Soc. Am. 68, 1820 (1980). 